ANNALES

POLONICI MATHEMATICI

XIII (1963)

\title{
Continuous solutions of a functional equation *
}

by M. K. FonT, Jr. (Athens, Georgia)

\$ 1. In a series of two papers, [2] and [3], M. Kuczma has studied the functional equation

$$
\varphi(\varphi(x))=g(x, \varphi(x))
$$

where $\varphi$ denotes an unknown function and $g$ is given.

We assume that $\beta$ is a continuous and strictly increasing real valued function on the closed interval $[a, b]$ of real numbers such that $\beta(a)=a$, $\beta(b)=b$ and $\beta(x)>x$ for $a<x<b$. Concerning the function $g$, it is assumed that:

(i) $g(x, y)$ is defined for all points $(x, y)$ in the set $K=\{(x, y) \mid a \leqslant x \leqslant b$ and $x \leqslant y \leqslant \beta(x)\}, g$ is continuous on $K$, and $g$ is strictly increasing with respect to each variable;

(ii) $g(a, a)=a, g(b, b)=b, g(x, x)>x$ for $a<x<b$, and $g(x, y)>y$ for $a<x<b$ and $x<y<\beta(x)$;

(iii) $g(x, \beta(x))=\beta(x)$ for $a \leqslant x \leqslant b$.

(Our notation differs slightly from that of Kuczma, and we do not permit $a, b$ or $\beta(x)$ to be infinite.)

In his first paper, [2], Kuczma proves that if $a<x_{0}<b$, then equation (1) has solutions which are continuous and strictly increasing on $\left[x_{0}, b\right]$. In the second paper, [3], he proves that equation (1) has a solution which is defined and increasing on $[a, b]$, but he does not prove that such a solution exists which is continuous. Kuczma raises the question in [3] as to whether (1) has a continuous solution which is defined on $[a, b]$. He conjectures that (1) has a unique solution defined in $[a, b]$, and points out that if this is true then the unique solution must be continuous and strictly increasing.

We show in this paper that although (1) always does have a solution which is continuous and strictly increasing on $[a, b]$, such a solution is not necessarily unique.

* This work was supported by a National Science Foundation Grant, NSFG12972. 
\$ 2. Let $C$ be the set of all real valued continuous functions on $[a, b]$. If $\varphi \in C$ and $\psi \in C$, we define

$$
\varrho(\varphi, \psi)=\sup \{|\varphi(x)-\psi(x)| \mid a \leqslant x \leqslant b\} .
$$

It is well known that $\varrho$ is a metric for $C$, and that convergence of sequences in $(C, \varrho)$ is equivalent to uniform convergence.

As a matter of notational convenience, we assume that "function" has been defined by the "set of ordered pairs" method. Thus, a member $\varphi$ of $C$ is the same as the subset of the plane which is frequently called "the graph of $\varphi$ ".

We define

$$
M=\{\varphi \mid \varphi \in C, \varphi \subset K \text { and } \varphi \text { is strictly increasing }\},
$$

and then define a function $T$ on $M$ into $M$ by requiring that

$$
T(\varphi)(x)=g\left(\varphi^{-1}(x), x\right)
$$

for all $\varphi \in M$ and $a \leqslant x \leqslant b$. It is easily seen that $T$ is one-to-one and continuous.

It is easy to see that a continuous and strictly increasing function $\varphi$ satisfies (1) if and only if $T(\varphi)=\varphi$. Hence solutions of our equation are fixed points for the mapping $T$.

As in [3], we define a homeomorphism $R$ on $K$ into $K$ by letting

$$
R(x, y)=(y, g(x, y)) \text {. }
$$

If $p=(x, y)$, it is notationally convenient to write $R(p)$ for $R(x, y)$.

Remark 1. It is easy to verify that if $p \in K$ and $\varphi \in M$, then $p \in \varphi$ if and only if $R(p) \in T(\varphi)$.

LEMMA 1. If $\varepsilon>0$, there exists $\delta>0$ such that if $a \leqslant u<v \leqslant b$, $v-u \geqslant \varepsilon$ and $\varphi \in T[M]$, then $(\varphi(v)-\varphi(u)) /(v-u) \geqslant \delta$.

Proof. Let us assume the lemma false. Then, for each positive integer $n$ there would exist $\varphi_{n} \in T[M]$ and numbers $u_{n}$ and $v_{n}$ such that $a \leqslant u_{n}<v_{n} \leqslant b, v_{n}-u_{n} \geqslant \varepsilon$ and $\left(\varphi\left(v_{n}\right)-\varphi\left(u_{n}\right)\right) /\left(v_{n}-u_{n}\right)<1 / n$. If we let $U_{n}=\left(u_{n}, \varphi_{n}\left(u_{n}\right)\right)$ and $V_{n}=\left(v_{n}, \varphi_{n}\left(v_{n}\right)\right)$, we may assume without loss of generality that there are points $U_{0}=\left(u_{0}, y\right)$ and $\nabla_{0}=\left(v_{0}, y\right), u_{0}<v_{0}$, such that $U_{n} \rightarrow U_{0}$ and $V_{n} \rightarrow V_{0}$ as $n \rightarrow \infty$. If $x_{1}, x_{2}, y_{1}, y_{2}$ are numbers such that $\left(x_{1}, y_{1}\right)=R^{-1}\left(U_{0}\right)$ and $\left(x_{2}, y_{2}\right)=R^{-1}\left(\nabla_{0}\right)$, then $x_{1}>x_{2}$ and $y_{1}<y_{2}$. This is impossible, since $R^{-1}\left(U_{n}\right) \rightarrow R^{-1}\left(U_{0}\right)$ and $R^{-1}\left(V_{n}\right) \rightarrow R^{-1}\left(V_{0}\right)$ as $n \rightarrow \infty$, and by our Remark $R^{-1}\left(U_{n}\right)$ and $R^{-1}\left(V_{n}\right)$ are on the graph of the increasing function $T^{-1}\left(\varphi_{n}\right)$ for each $n$.

Corollary 1. $\overline{T[M]} \subset M$.

Proof. Let $\varphi \epsilon \overline{T[\bar{M}]}$. We must prove that $\varphi$ is strictly increasing. There is a sequence $\varphi_{1}, \varphi_{2}, \varphi_{3}, \ldots$ in $T[M]$ which converges uniformly to $\varphi$. 
Suppose $a \leqslant u<v \leqslant b$. Let $\varepsilon=(v-u) / 2$ and use Lemma 1 to obtain $\delta>0$ so that $\left(\varphi_{n}(v)-\varphi_{n}(u)\right) /(v-u)>\delta$ for all $u$. It follows that $(\varphi(v)-$ $-\varphi(u)) /(v-u) \geqslant \delta$ and hence $\varphi$ is strictly increasing.

LEMMA 2. $T^{2}[M]$ is an equi-uniformly continuous set of functions.

Proof. Suppose that $T^{2}[M]$ is not an equi-uniformly continuous set of functions. Then there exists a sequence $\psi_{1}, \psi_{2}, \psi_{3}, \ldots$ of functions in $T^{2}[M]$ and points $p_{n}$ and $q_{n}$ in $\psi_{n}$ such that $p_{n} \rightarrow p$ and $q_{n} \rightarrow q$ as $n \rightarrow \infty$, where $p$ and $q$ are distinct points in $R^{2}[K]$ which have the same first coordinate. Thus, $R^{-1}\left(p_{n}\right) \rightarrow R^{-1}(p)$ and $R^{-1}\left(q_{n}\right) \rightarrow R^{-1}(q)$ as $n \rightarrow \infty$, where $R^{-1}\left(p_{n}\right)$ and $R^{-1}\left(q_{n}\right)$ are points on $T^{-1}\left(\psi_{n}\right)$ and $R^{-1}(p)$ and $R^{-1}(q)$ are distinct points of $R[K]$ which have the same second coordinate. Since $T^{-1}\left(\psi_{n}\right) \in T[M]$ for each $n$, this is not possible by Lemma 1 .

COROLLARY 2. $\overline{T^{2}[M]}$ is a compact subset of $T[M]$.

Remark 2. If we partially order $M$ in the natural way by defining $a_{1} \leqslant a_{2}$ if and only if $a_{1}(t) \leqslant a_{2}(t)$ for all $t \in[a, b]$, then $T$ is order reversing on $M$. That is, if $\alpha_{1} \leqslant a_{2}$ then $T\left(a_{2}\right) \leqslant T\left(a_{1}\right)$.

THEOREM 1. There exists a continuous, strictly increasing solution of (1) which is defined in the closed interval $[a, b]$.

Proof. Let $\psi_{0}=\beta$ and $\psi_{n}=T\left(\psi_{n-1}\right)$ for $n>0$. It is easily seen that $\psi_{1}$ is the identity function on $[a, b]$, and hence $\psi_{0}$ and $\psi_{1}$ are respectively the "largest" and "least" members of. $M$. By repeated application of Remark 2, we obtain: $\psi_{0} \geqslant \psi_{2} \geqslant \psi_{4} \geqslant \ldots ; \psi_{2 n} \geqslant \psi_{2 n+1}$ for each $n$; and $\psi_{1} \leqslant \psi_{3} \leqslant \psi_{5} \leqslant \ldots$ It follows that the sequence $\psi_{0}, \psi_{2}, \psi_{4}, \ldots$ converges pointwise to a monotone increasing function $\sigma$, and that $\psi_{1}, \psi_{3}, \psi_{5}, \ldots$ converges pointwise to a monotone increasing function $\tau$. It is obvious that $\tau \leqslant \sigma$. Since $\psi_{n} \in T^{2}[M]$ for $n>1$, the functions in the preceding sequences are equi-uniformly continuous, and hence pointwise convergence implies uniform convergence. Thus, $\tau$ and $\sigma$ are continuous functions, $\lim _{n \rightarrow \infty} \varrho\left(\psi_{: n}, \sigma\right)=0$ and $\lim _{n \rightarrow \infty} \varrho\left(\psi_{2 n+1}, \tau\right)=0$. Since $\psi_{n} \in T[M]$ for $n>0$, we see that $\sigma$ and $\tau$ belong to $\overline{T[M]}$, and it follows from Corollary 1 that $\sigma$ and $\tau$ belong to $M$.

We now define $K_{0}=K$ and $K_{n}=R\left[K_{n-1}\right]$ for $n>0$. It is easily seen that

$$
\begin{aligned}
K_{2 n} & =\left\{(x, y) \mid a \leqslant x \leqslant b \text { and } \psi_{2 n+1}(x) \leqslant y \leqslant \psi_{2 n}(x)\right\} \\
K_{2 n+1} & =\left\{(x, y) \mid a \leqslant x \leqslant b \text { and } \psi_{2 n+1}(x) \leqslant y \leqslant \psi_{2 n+2}(x)\right\} .
\end{aligned}
$$

If we let $D=\bigcap_{n=0}^{\infty} K_{n}$, then it follows that

$$
D=\{(x, y) \mid a \leqslant x \leqslant b \text { and } \tau(x) \leqslant y \leqslant \sigma(x)\} .
$$

For a point $p \in K, R^{n}(p)$ is defined for all negative integers $n$ if and only if $p \in D$. (We observe that, in the notation of [3], $V_{s}=[\tau(x), \sigma(x)]$.) 
Now, choose any point $P_{0}=\left(x_{0}, y_{0}\right)$ in $D$ for which $a<x_{0}<b$, and let $P_{1}=\left(x_{1}, y_{1}\right)=R\left(P_{0}\right)$. Define $\varphi$ on $\left[x_{0}, x_{1}\right]$ so as to be continuous and strictly increasing, and so as to satisfy $\varphi\left(x_{0}\right)=y_{0}, \varphi\left(x_{1}\right)=y_{1}, \tau(x) \leqslant \varphi(x)$ $\leqslant \sigma(x)$ for $x_{0} \leqslant x \leqslant x_{1}$. It is possible to do this since $\sigma$ and $\tau$ are continuous and strictly increasing. Finally, as was shown in [2], [3], $\varphi$ may be extended uniquely to a function on $[a, b]$ which satisfies equation (1). The solution of (1) which we obtain in this manner is continuous and strictly increasing on $[a, b]$.

\$3. In this section we prove an extension theorem concerning functions which are strictly increasing in each variable. This result is applied in the final section of our paper to show that equation (1) does not necessarily have a unique solution.

If $p=\left(p_{1}, p_{2}\right)$ and $q=\left(q_{1}, q_{2}\right)$ are points in the plane, we define $p<q$ to mean that $p_{1} \leqslant q_{1}, p_{2} \leqslant q_{2}$ and $p \neq q$. If $f$ is a real valued function whose domain is a subset of the plane, we say that $f$ is increasing if $f(p)$ $\leqslant f(q)$ whenever $p<q$, and we say that $f$ is strictly increasing if $f(p)<f(q)$ whenever $p<q$.

We let $s$ be a positive number, and let $S$ be the solid square defined by

$$
S=\{(x, y) \mid-s \leqslant x \leqslant s \text { and }-s \leqslant y \leqslant s\} \text {. }
$$

Although the following lemma could be obtained as a special case of a theorem due to L. E. Ward, Jr. (see [4], p. 365, Theorem 11), it seems advisable to give a separate proof.

LEMra 3. If $A$ is a closed subset of $S$ and $f$ is a continuous and increasing real valued function on $A$, then $f$ can be extended to a continuous and increasing real valued function $g$ in $S$.

Proof. There is no loss of generality in assuming that $(-s,-s)$ and $(s, s)$ are members of $A$. We now define two real valued functions $U$ and $L$ on $S$ by letting

and

$$
U(p)=\inf \{f(z) \mid z \in A \text { and } p \leqslant z\}
$$

$$
L(p)=\sup \{f(z) \mid z \epsilon A \text { and } z \leqslant p\}
$$

for all $p \in S$. The following properties of $U$ and $L$ are easily verified:

(i) $L(p) \leqslant U(p)$ for all $p \in S$,

(ii) $L$ and $U$ are increasing on $s$,

(iii) $U$ is lower semi continuous and $L$ is upper semi continuous on $S$, and

(iv) if $p \in A$, then $L(p)=f(p)=U(p)$.

It is well known that there is a continuous real valued function $h$ on $S$ such that $L(p) \leqslant h(p) \leqslant U(p)$ for all $p \in S$ (see [1], p. 75, the remark after Theorem 9). If we now define

$$
g(p)=\sup \{h(z) \mid z \in S \text { and } z \leqslant p\},
$$


we obtain a continuous and increasing function $g$ on $\mathcal{S}$, and $L(p) \leqslant g(p)$ $\leqslant U(p)$. It follows that $g$ is the desired extension of $f$.

THEOREM 2. If $A$ is a closed subset of $S$ and $f$ is a continuous and strictly increasing real valued function on $A$, then $f$ can be extended to a continuous and strictly increasing real valued function $g$ on $S$.

Proof. We may assume without loss of generality that $(-s,-s)$ and $(s, s)$ are members of $A$. Functions $U$ and $L$ are defined as in the proof of Lemma 3. Since $h$ is continuous and strictly increasing on $A$, it follows that $L(p)<U(p)$ for $p \in S-A$. We define $m(p)=[L(p)+$ $+U(p)] / 2$ and $\varepsilon(p)=[U(p)-L(p)] / 4$ for $p \in S-A$. Moreover, for $p=\left(p_{1}, p_{2}\right) \in S-A$ and $z=\left(z_{1}, z_{2}\right) \in S$, we define $\mu(p, z)=m(p)+$ $+\left(z_{1}-p_{1}\right)+\left(z_{2}-p_{2}\right)$. Since $U$ is lower semi continuous and $L$ is upper semi continuous, there exists $\delta(p)>0$ for each $p \in S-A$ such that if $z \in S$ and $|z-p| \leqslant \delta(p)$, then

$$
\begin{aligned}
L(z)<L(p)+\varepsilon(p) & =m(p)-\varepsilon(p) \\
& <\mu(p, z)<m(p)+\varepsilon(p)=U(p)-\varepsilon(p)<U(z) .
\end{aligned}
$$

If we define $B(p)=S \cap\{z|| z-p \mid \leqslant \delta(p)\}$ for $p \in S-A$, then $B(p)$ is a closed set and $B(p) \cap A=\emptyset$. There exists a sequence $q_{1}, q_{2}, q_{3}, \ldots$ of points in $S-A$ such that

$$
S-A=\bigcup_{n=1}^{\infty} B\left(q_{n}\right)
$$

We now define a function $f_{n}$ on $A \cup B\left(q_{n}\right)$ by letting

$$
f_{n}(z)=\left\{\begin{array}{lll}
f(z) & \text { for } & z \in A \\
\mu\left(q_{n}, z\right) & \text { for } & z \in B\left(q_{n}\right)
\end{array}\right.
$$

Each $f_{n}$ is easily seen to be strictly increasing on $A \cup B\left(q_{n}\right)$, and we use Lemma 3 to extend $f_{n}$ to a function $g_{n}$ which is continuous and increasing on $s$. The functions $g_{n}$ are uniformly bounded since $(-8,-s)$ and $(s, s)$ are in $A$ and each $g_{n}$ assumes its minimum and maximum respectively at these points. We now define:

$$
g(z)=\sum_{n=1}^{\infty} g_{n}(z) / 2^{n}
$$

for each $z \in S$. The function $g$ is a continuous extension of $f$ and is easily shown to be strictly increasing on $S$.

\$ 4. In this paragraph we construct a function $g$ for which the equation (1) will have more than one solution. This is done by first describing a pair of functions $\varphi$ and $\psi$ and then using Theorem 2 to obtain a function $g$ for which (1) will have $\varphi$ and $\psi$ as solutions. 
Let $[a, b]$ be a closed interval, and let $\varphi$ be a homeomorphism of $[a, b]$ onto itself such that $\varphi(x)>x$ for $a<x<b$. We choose a point $p$ interior to $[a, b]$, and then define $p_{n}=\varphi^{n}(p)$ for each integer $n$. It is obvious that: $p_{n}<p_{n+1}$ for each $n, p_{n} \rightarrow b$ as $n \rightarrow \infty$, and $p_{n} \rightarrow a$ as $n \rightarrow-\infty$. We let $J_{n}=\left[p_{n}, p_{n+1}\right]$.

We now define, for each integer $n$, an orientation preserving homeomorphism $\psi_{n}$ on $J_{n}$ onto $J_{n+1}$. First, we choose $\psi_{0}$ to be a homeomorphism of $J_{0}$ onto $J_{1}$ such that $\psi_{0}(x)>\varphi(x)$ for $p_{0}<x<p_{1}$. If $n$ is a non negative integer and $\psi_{-n}, \psi_{-n+1}, \ldots, \psi_{n}$ have been defined, we define

$$
\psi_{n+1}(x)=\left[\varphi(x)+\varphi^{2} \psi_{n}^{-1}(x)\right] / 2
$$

and we choose a homeomorphism $\psi_{-n-1}$ of $J_{-n-1}$ onto $J_{-n}$ such that

and

$$
\psi_{-n-1}(x)<\psi_{-n}^{-1} \varphi^{2}(x) \quad \text { if } n \text { is even }
$$

$$
\psi_{-n-1}(x)>\psi_{-n}^{-1} \varphi^{2}(x) \quad \text { if } n \text { is odd . }
$$

We now define a homeomorphism $\psi$ of $[a, b]$ onto $[a, b]$ by letting $\psi(a)=a, \psi(b)=b$, and $\psi(x)=\psi_{n}(x)$ for $x \in J_{n}$. It is not difficult to prove (although there are several cases to consider) that for each integer $n$, if $p_{n}<x<p_{n+1}$ then:

and

$$
\psi(x)>\varphi(x) \quad \text { and } \quad \psi^{2}(x)>\varphi^{2}(x) \quad \text { if } n \text { is even, }
$$

$$
\psi(x)<\varphi(x) \quad \text { and } \quad \psi^{2}(x)<\varphi^{2}(x) \quad \text { if } n \text { is odd . }
$$

It is easy to see that

$$
\max (\varphi(x), \psi(x))<\min \left(\varphi^{2}(x), \psi^{2}(x)\right)
$$

for $a<x<b$, and hence we may choose a homeomorphism $a$ on $[a, b]$ onto itself such that

$$
\max (\varphi(x), \psi(x))<a(x)<\min \left(\varphi^{2}(x), \psi^{2}(x)\right)
$$

for $a<x<b$. We choose a homeomorphism $\beta$ on $[a, b]$ onto itself such that

for $a<x<b$.

$$
\max \left(\varphi^{2}(x), \psi^{2}(x)\right)<\beta(x)
$$

We now define a set $A$ in the plane by

$$
A=\beta \cup \varphi \cup \psi \cup\{(x, x) \mid a \leqslant x \leqslant b\} .
$$

We define a real valued function $f$ on $A$ by requiring:

$$
f(x, \beta(x))=\beta(x), \quad f(x, \varphi(x))=\varphi^{2}(x), \quad f(x, \psi(x))=\psi^{2}(x),
$$

and $f(x, x)=\alpha(x)$. It is easy to verify that $f$ is strictly increasing on $A$. 
We define $K=\{(x, y) \mid a \leqslant x \leqslant b$ and $x \leqslant y \leqslant \beta(x)\}$. We may now enclose $K$ in a sufficiently large square and apply Theorem 2 to obtain a function $g$ on $K$ which is continuous, strictly increasing, and an extension of $f$. Since

$$
\varphi^{2}(x)=f(x, \varphi(x))=g(x, \varphi(x))
$$

and

$$
\psi^{2}(x)=f(x, \psi(x))=g(x, \psi(x)),
$$

we see that both $\varphi$ and $\psi$ are solution of (1).

\section{References}

[1] J. Dieudonné, Ore généralisation des espaces compacts, Journal de Mathematiques Pures et Appliquées 23 (1944), p. 65-76.

[2] M. Kuczma, On monotonic solutions of a functional equation, I, Ann. Polon. Math. 9 (1960), p. $295-297$.

[3] - On monotonic solutions of a functional equation, II, Ann. Polon. Math. 10 (1961), p. 161-166.

[4] L. E. Ward, Jr., Binary relations in topological spaces, Anais da Academia Brasileira de Ciências 26 (1954), p. 357-373.

THE UNIVERSity OF GEORGIA, ATHENS, GEORGIA (USA)

Reşu par la Rédaction le 5. 1. 1962 